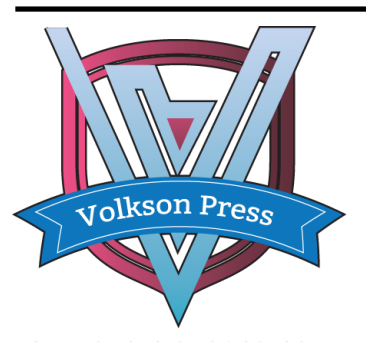

Contents List available at VOLKSON PRESS

New Materials and Intelligent Manufacturing (NMIM)

DOI : http://doi.org/10.26480/icnmim.01.2018.49.53

Journal Homepage: https://topicsonchemeng.org.my/

ISBN: 978-1-948012-12-6

\title{
SEPARATION OF DIISO-PROPYL ETHER+ISO-PROPANOL+N-PROPANOL BY EXTRACTIVE DISTILLATION AND PRESSURE-SWING DISTILLATION: SIMULATION AND ECONOMIC OPTIMIZATION
}

\author{
Wang Gui-ying, Liu Yan-jie, Chen Li \\ Collage of Petro-chemical Technology, Jilin Institute of Chemical Technology Chengde Street 45, JiLin, China. \\ *Corresponding Author email: wgy715@163.com
}

This is an open access article distributed under the Creative Commons Attribution License, which permits unrestricted use, distribution, and reproduction in any medium, provided the original work is properly cited

\section{ARTICLE DETAILS}

Article History:

Received 26 June 2018

Accepted 2 July 2018

Available online 1 August 2018

\section{ABSTRACT}

We have studied, simulated and evaluated economically two separation alternatives of a ternary mixture made up of $25 \mathrm{~mol} \%$ diisopropyl ether, $60 \mathrm{~mol} \%$ isopropyl alcohol and $15 \mathrm{~mol} \% \mathrm{n}$-propyl alcohol by means of a practical case of a plant to treat $100 \mathrm{kmol} / \mathrm{h}$. The simulation has been carried out satisfactorily by mean of a package of commercial software Aspen Plus using the thermodynamic model NRTL-RK. The extractive distillation using N,N-dimethyl acetamide as a solvent and pressure-swing distillation have been optimized independently and the best configurations have been evaluated economically. Results show that, for this particular case, the pressure-swing distillation is more attractive.

\section{KEYWORDS}

DIPE, IPA, NPA, ED, SPD, simulation and economic optimization.

\section{INTRODUCTION}

The addition of oxygenated compounds, such as ethers and alcohols, to gasoline has been of interest owing to their pollution reducing and octaneenhancing capabilities. Diisopropyl ether (DIPE) has become an important gasoline additive over the past decade and it is also widely used in many other fields, such as tobacco production and synthetic chemistry [1]. Iso propyl alcohol (IPA) and n-propyl alcohol (NPA) are extensively used in pharmaceutical industry as a chemical intermediate and solvent [2]. IPA can be produced by using solid acid or liquid acid as catalytic agent with DIPE and NPA as a byproducts [3]. The separation of ternary system of DIPE+IPA+NPA is the key downstream process that determines the entire process economic benefits. However, IPA+DIPE, or DIPE+NPA couled not be separated by conventional distillation process because they form a binary minimum boiling homogeneous azeotropes separately at different pressure [1,2].

There are many techniques suitable for separation of azeotropic mixtures, such as pressure swing distillation (PSD), extractive distillation (ED), azeotopic distillation, and some new coupling separation techniques and so on [1-3]. Among the various techniques available for breaking azeotropes, how to select a suitable one, design and optimize the separation sequences are a complex task.

ED is the common method for the separation of azeotropic mixture adding a solvent (entrainer) that is capable of strongly modifying the relative volatility of the mixture in large scale productions. The synthesis and design of extractive distillation processes take place in two steps. The first one involves the selection of one or more candidate solvents, and the choice of one or more column configurations. The second step, process design, involves the search for optimal process parameter values. The success of the second step depends on the solutions obtained for the first one because efficiency in extractive distillation is largely determined by the choice of a suitable entrainer. Based on the guidelines for the solvent screening, initially, three solvents: 2-ethoxyethanol (ECS), 2butoxyethanol (BCS) and N,N-dimethyl acetamide (DMA) have been chosen. Therefore, in order to be able to select the best solvent, we have carried out simulations with Aspen Plus. Once the solvent has been selected, we have designed the separation sequence and optimized the operating parameters.

On the other hand, to investigate how the pressure-swing distillation
(PSD) works with the DIPE+IPA, DIPE+NPA azeotropic system, we have done a simulation of the vapor-liquid equilibrium (VLE) using Aspen Plus 8.4 at different pressures. The azeotrope of DIPE- IPA, DIPE-NPA are very sensitive to pressure, therefore, the system in our case can be separated by pressure swing distillation $[4,5]$.

The aim of this work is to study the influence of the operation variable values and column configuration on the performance of the DIPE+IPA+NPA separation by extractive distillation and by swingpressure distillation, with the help of a commercial simulator Aspen Plus. Finally, we have chosen the best alternative process for the separation of the ternary mixture under study from the economic point of view.

\section{SIMULATION}

\subsection{Problem definition}

The two alternatives considered in this study (PSD and ED) were simulated starting from the same initial data. The feed is a mixture made up of $25 \mathrm{~mol} \%$ of DIPE, $60 \mathrm{~mol} \%$ of IPA and $15 \mathrm{~mol} \%$ of NPA, with a flow rate of $100 \mathrm{kmol} / \mathrm{h}$, column operation conditions providing necessary mass purity of products are $\geq 99.8 \%$. We took 8400 working hours per year, payback period 3 years.

\subsection{Property package}

The accuracy of the simulated results is strongly dependent on the quality of the binary parameters for the liquid-phase activity coefficient models. In this paper, the simulation is undertaken with Aspen Plus 8.4, NRTL-RK activity model is chosen because it is the most suitable.

\subsection{Economic model}

Economic analysis is the crucial factor in a steady state comparison. Total annual cost (TAC) [6] that includes capital investment and operating cost, is usually considered as the objective function for evaluating the distillation process. The capital investment includes column vessels and heat exchangers. Additional costs, including pumps, reflux drums, valves and pipes can be ignored because their costs are much lower than those of the column vessels and heat exchangers. 


\section{DETERMINATION OF SEPARATION PROCESS}

\subsection{Analysis to residual curve for DIPE+IPA+NPA ternary system}

The residual curve diagram of DIPE, IPA and NPA at $100 \mathrm{kPa}$ is obtained through Aspen Plus as shown in Figure 1. In Figure 1, A is the azeotropic point formed by DIPE and IPA at $65.57{ }^{\circ} \mathrm{C}$ with $77.36 \mathrm{~mol} \%$ of DIPE and $22.64 \mathrm{~mol} \%$ of IPA at atmospheric pressure. Figure 1 displays the essential features of Serafimov's class 1.0-1a [7]. DIPE (1) and IPA (2) are both saddle points on the residue curve map (RCM) and form a minimumboiling azeotrope (A), it is a RCM unstable node. The original feed composition (F) is in Figure 1 with $25 \mathrm{~mol} \%$ of IPA, $60 \mathrm{~mol} \%$ of IPA and $15 \mathrm{~mol} \%$ of NPA. In a traditional way, pure NPA $\left(B_{1}\right)$ is obtained from the bottom of the preliminary distillation column (PDC), while the mixture of DIPE and IPA $\left(D_{1}\right)$ is usually obtained from the top, the splitting line of PDC is $\mathrm{B}_{1}-\mathrm{F}-\mathrm{D}_{1}$.

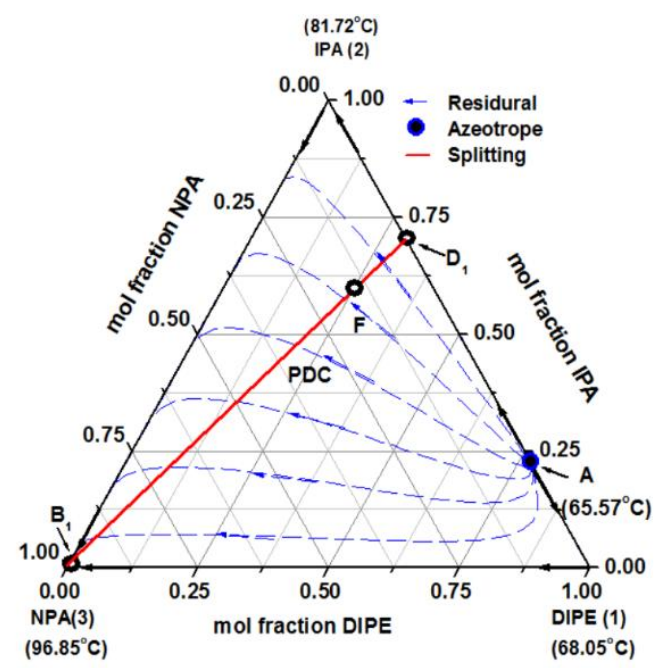

Figure 1: Residue curve map plot at $100 \mathrm{kPa}$ for ternary systems DIPE(1)+IPA(2)+NPA(3).

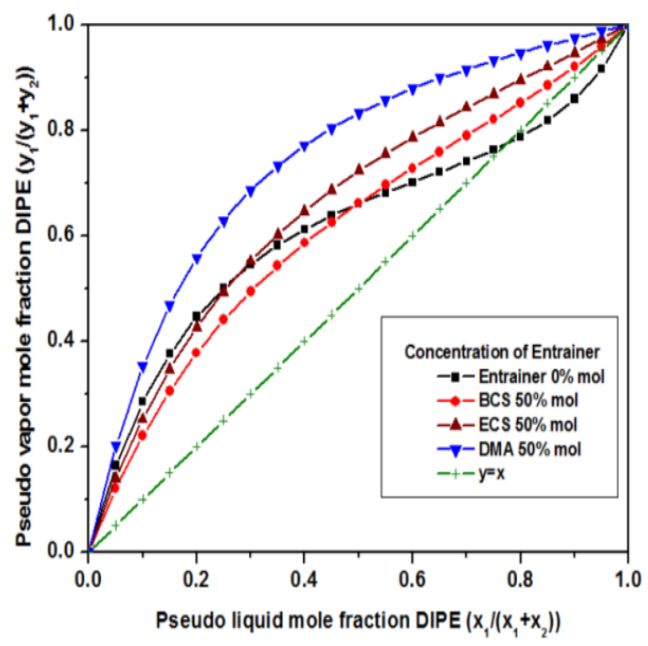

Figure 2: Pseudo binary VLE of DIPE(1)+IPA(2) in presence of BCS, ECS and DMA.

\subsection{Extractive distillation}

\subsubsection{Solvent selection}

Since the solvent is the core of extractive distillation, more attention should be paid on the selection of potential solvents. The extractive agent should have the following characteristics: lower volatility than the volatilities of the mixture components, increases the relative volatility of the mixture, preferably does not form an azeotrope with any of the mixture components, and is readily available and cheap ${ }^{[8]}$. In order to verify the effect of the aforementioned solvents, the VLE of the ternary mixtures, on a solvent free basis, are simulated and plotted in Figure 2. As can be observed, ECS, BCS and DMA all eliminate the DIPE(1)+IPA(2) azeotrope, DMA is the best promising entrainer for the separation of DIPE and IPA azeotropic mixture by extractive distillation. In the natural way,
DIPE would be obtained as the overhead product and IPA together with the solvent (DMA) would be obtained as the bottom product.

\subsubsection{Sequencing of the extractive distillation process}

According to the results obtained above, the best solvent seems to be DMA, for the design and optimize of an extractive distillation system, the residual curve diagram of DIPE(1)+IPA(2)+DMA(3) is obtained through Aspen Plus as shown in Figure 3. Neither add new azeotropes nor form distillation boundaries, and the bubble point of DMA (3) is the highest, which confirm that DMA is excellent heavy entrainer. The light original component DIPE and the heavy original component IPA are both saddle points on the RCM and form a minimum-boiling azeotrope (A), which is a RCM unstable node, the heavy entrainer DMA is a RCM stable node.

Isovolatility curves are useful for determining the desirable product in the distillate stream of the ED, the isovolatility line $\left(\alpha_{12}=1\right)$ is presented for the binary system DIPE(1)+IPA(2) in presence of DMA. For ternary mixtures that if these isovolatility lines intersect the 1-3 (solvent) edge, component 1 is recovered as distillate in an extractive column (ED) while component 2 is recovered as distillate in the entrainer recovery column (SRC) [7], the splitting line of EDC and SRC are $D_{2}-M_{2}-B_{2}, B_{3}-B_{2}-D_{3}$ respectively in Figure 3.

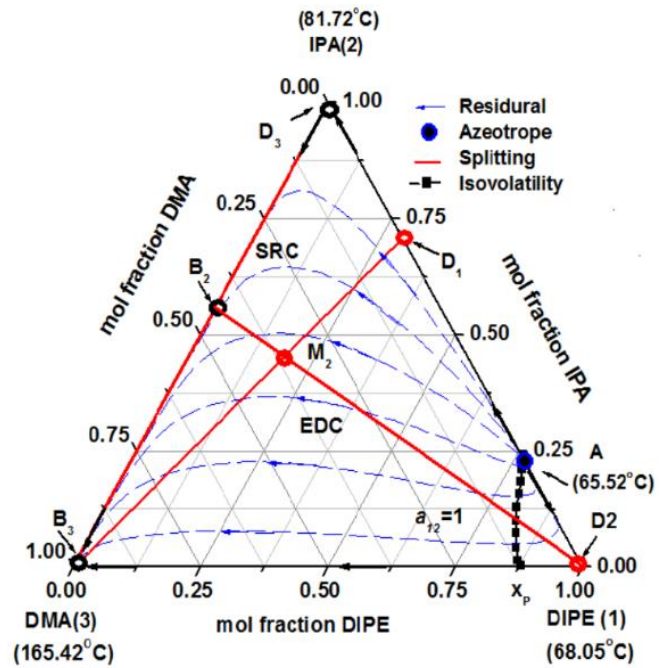

Figure 3: Residue curve map plot at $100 \mathrm{kPa}$ for ternary systems DIPEIPA-DMA

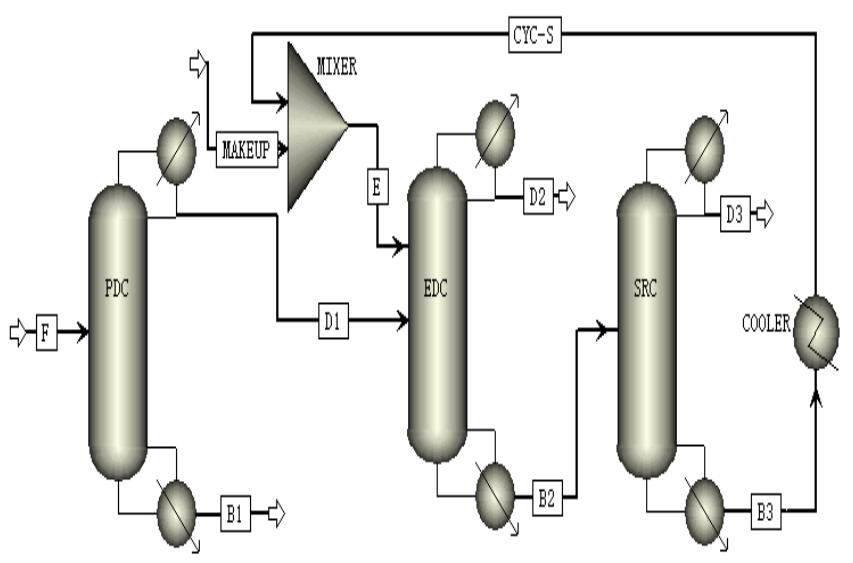

Figure 4: Separation process of DIPE(1)+IPA(2)+NPA(3) by ED.

\subsubsection{Separation process of DIPE+IPA+NPA by extractive distillation}

After the entrainer has been selected, in this work we have chosen the DMA, attention is directed to the sequence of the distillation column. The specific process is shown in Figure 4. PDC is the preliminary separating column, and its main purpose is to divide the ternary mixture into DIPE+IPA mixture $\left(D_{1}\right)$ and pure NPA $\left(B_{1}\right)$. The mixture of DIPE+IPA $\left(D_{1}\right)$ is then treated in the EDC, in which the solvent is added at the top trays of the EDC. In this column, DMA increases the volatility of DIPE with respect to IPA and thus makes the separation easier. Since DMA is much less volatile than either DIPE or IPA, it flows down the column to leave with the bottom product $\left(\mathrm{D}_{2}\right)$. The solvent recovery column (SRC) removes IPA from DMA, this is an easy separation because the solvent is much less 
volatile than DMA, pure DIPE $\left(\mathrm{D}_{3}\right)$ distillate from top of SRC, and the pure DMA $\left(\mathrm{B}_{3}\right)$ recover at bottom then cooled (CYS-S) and recycled back to the EDC. To balance the tiny entrainer losses in both the $\mathrm{D}_{2}$ and $\mathrm{D}_{3}$ streams, a small makeup stream of entrainer should be added.

\subsubsection{Optimization}

\subsubsection{ED Partial optimization}

In order to select the best conditions to carry out the global economical optimization, we start with a partial optimization specifying some variables and using the total reboiler duty as a reference variable. The variables chosen to be specified can be characterized as either design variables or optimization variables. Design variables are those whose values are set by market demands or physical conditions. In our study we specified the temperature, pressure, flow rate, composition of ternary feed and the purity of products DIPE, IPA and NPA. Finally, solvent makeup was chosen as a pure component at ambient conditions. (case studies) analyzing the variation of stage number and reboiler heat duty (RHD) as a function of the reflux ratio, in order to set the number of ideal trays and feed position for PC and SRC columns.

In the extractive column we cannot use the short-cut design facility, so we have studied five cases varying the number of ideal trays from 20 to 40 , selecting in each case the optimum solvent-to-feed ratio (S/F), reflux ratio, the best solvent and feed entry stage, in Table 1 the five cases are specified.

From previous simulation runs, it was found that the solvent-to-feed ratio is very critical, having a significant effect on the reflux flow rate in both columns of EDC and SRC, and therefore on the total reboiler heat duty, so the partial optimization was made over this variable. In each case, the solvent-to-feed ratio is then adjusted to minimize the total RHD needed for the process. As an example (ED-3), Figure 5 shows this effect. This procedure has been applied successfully for all five cases. Table 1 shows a summary of the partial optimization based on total reboiler heat duty as a reference variable.

With the short-cut design tool of Aspen we did a preliminary optimization

Table 1: Number of ideal trays and economic results in the ED for each case

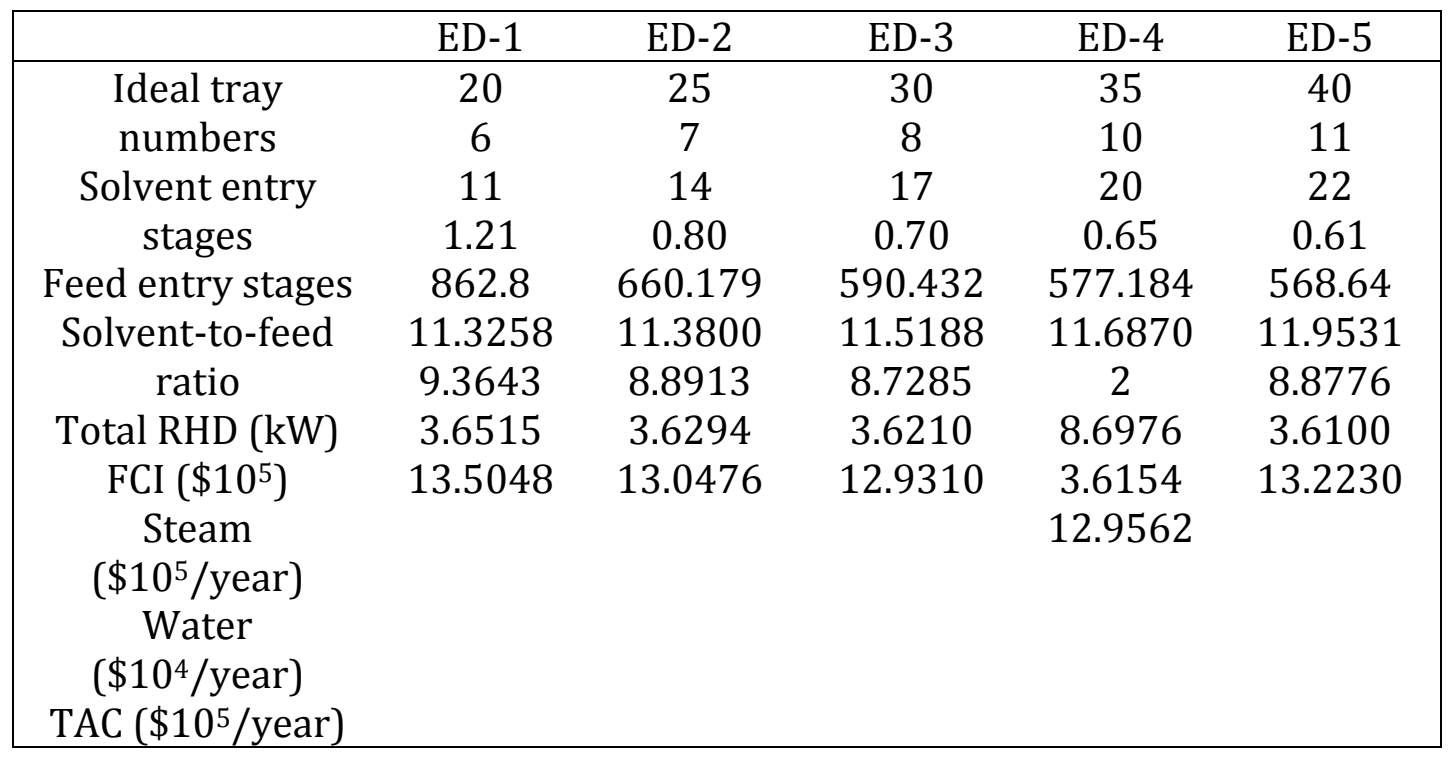

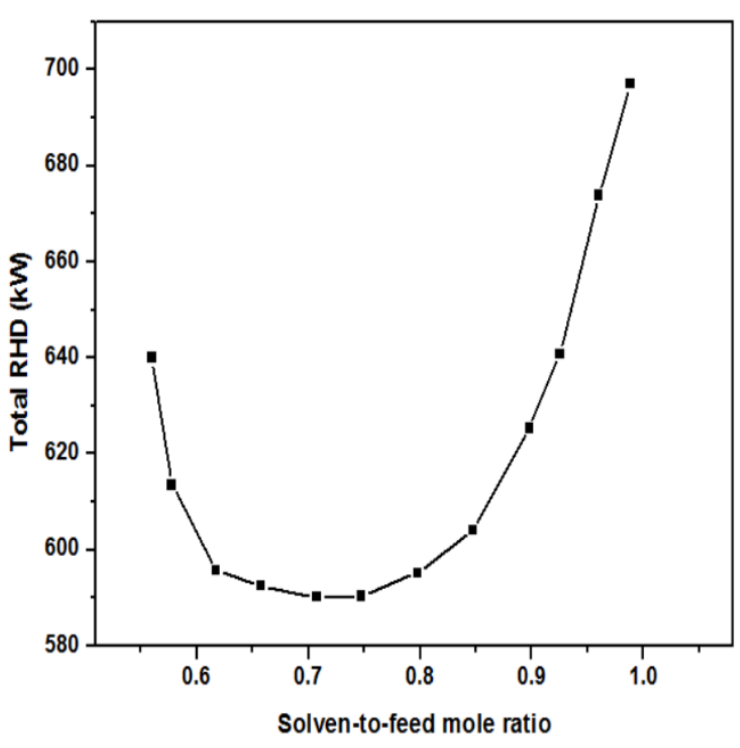

Figure 5: Total reboiler heat duty (RHD) vs. solvent-to-feed ratio (ED-3)

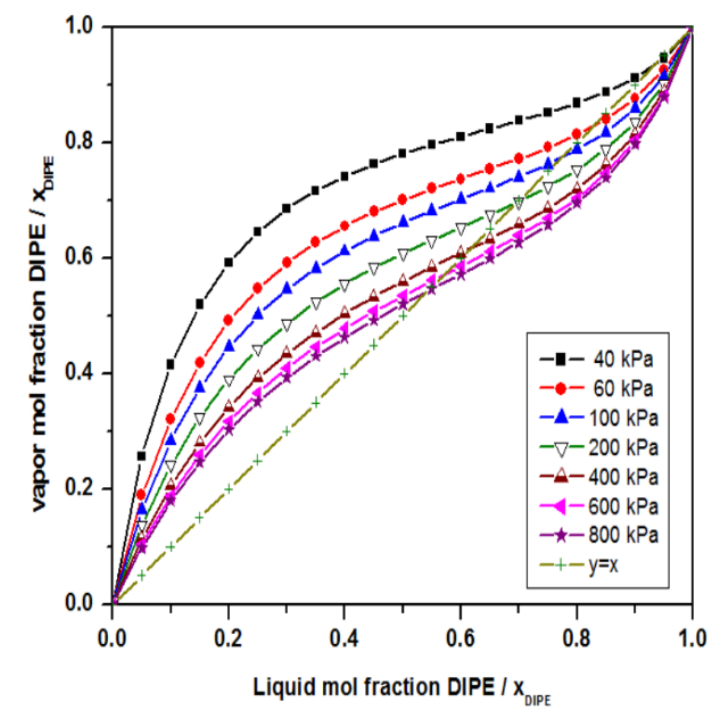

Figure 6: VLE for DIPE(1)+IPA(2) binary system at different pressures 


\subsubsection{ED global economic optimization}

As it can be seen in Table 1 the minimum reboiler heat duty corresponds to ED-5. However, this case requires a big number of stages in the extractive column, so, it would be an expensive column. Therefore, to determine the best of these five alternatives it is necessary to carry out an economic evaluation based on the minimum total annual costs (TAC), using the following objective function: $\mathrm{TAC}=\mathrm{FCI} /$ (payback period) + energy cost

Fixed capital cost (FCI), energy cost (water and steam), and TAC for the five cases also show in Table 1 . As it can be observed, there is an optimum condition for ED-3 (solvent-to-feed ratio = 0.7).

\subsection{Pressure-swing distillation}

It is well known that, in some cases, changing the system pressure can affect the VLE of a mixture. This effect can be used to separate a binary mixture containing a minimum boiling azeotrope, provided that this mixture significantly changes composition over a moderate pressure range.

To investigate the pressure sensitivity of DIPE(1)+IPA(2) azeotropic mixture, we begin with a simulation of the VLE at different pressures, using NRTL-RK thermodynamic model shown in Figure 6. We can observe a significant pressure influence on the azeotropic composition, the shift in the composition from $86.75 \%$ to $53.18 \mathrm{~mol} \%$ of DIPE as pressure from 40 $\mathrm{kPa}$ changes to $800 \mathrm{kPa}$. Therefore, the pressure-swing distillation could be a useful technique in order to overcome the binary azeotrope of DIPE and IPA.

\subsubsection{Operating pressures selection}

The actual operating pressure should be chosen within such a range that water can be used as coolant for the overhead condenser and steam can be used as a heating medium for the reboiler. According to that, the lowpressure column (LPC) will work at $60 \mathrm{kPa}$ and the high-pressure column (HPC) at $600 \mathrm{kPa}$.

\subsubsection{Sequencing of the pressure-swing distillation process}

For a binary mixture presenting a pressure-sensitive minimum boiling point azeotrope, the separation sequence is formed by two columns operating at different pressures. In our case, the separation sequence works as shown in Figure 7, where it is represented the Txy diagram for this DIPE+ IPA azeotropic mixture for both pressures, $60 \mathrm{kPa}$ and $600 \mathrm{kPa}$. The preliminary distillation column (PDC) distillate $\left(\mathrm{D}_{1}\right)$, is mixed with the recycled stream $\left(D_{3}\right.$ comes from the HPC top) to form the feed stream $\left(F_{2}\right)$, to the LPC, which operates at $60 \mathrm{kPa}$. Because $\mathrm{F}_{2}$ lies to the left of the azeotrope at $60 \mathrm{kPa}$, pure IPA can be obtained as a LPC bottom product $\left(\mathrm{B}_{2}\right)$, and a mixture near the azeotropic composition at $60 \mathrm{kPa}$ is the distillate $\left(D_{2}\right)$. Stream $D_{2}$ is the feed stream to the HPC, in this case at 600 $\mathrm{kPa}$. Because $\mathrm{F}_{3}\left(=\mathrm{D}_{2}\right)$ now lied to the right of the azeotrope at $600 \mathrm{kPa}$, the other pure component DIPE, can recovered in the HPC bottom product stream $\left(B_{3}\right)$, and a near azeotropic mixture becomes the distillate $\left(D_{3}\right)$, for recycling to the LPC, the specific process of pressure-swing distillation shown in Figure 8.

\subsubsection{PSD optimization}

Similar to the ED process, a partial optimization specifying some variables and using the total reboiler duty as a reference variable. For the PSD process, the optimization variables were the number of trays in each column, the recycle flow rate and the LPC distillate composition. Their values are subject to change as we proceed from the base case to the optimal design.

From the results of these case studies we selected 23 ideal trays (feed entry at stage 15) for the LPC and 25 ideal trays (feed entry at stage 15) for the HPC. To carry out the optimization, we studied five cases varying the LPC distillate composition ( $\mathrm{X}_{\mathrm{D} 2, \mathrm{DIPE}}$ ) from 0.78 to 0.82 molar fraction DIPE, selecting in each case the best recycle flow rate $\left(D_{3}\right)$ that minimizes the total reboiler heat duty needed for LPC and HPC both columns of PSD process. Figure 9 shows the variation of the total reboiler heat duty vs. the LPC distillate composition where it can be seen a clear minimum for the PSD process corresponds to 0.81 mole fraction of DIPE of the LPC column. This optimum is based on the minimum reboiler heat duty but it should correspond with the global economic optimum, since, having previously fixed the number of trays in each column, the operation costs (mainly the total reboiler heat duty) clearly dominate the total costs, for PSD-2 case, the capital investment, operating cost and TAC results also shown in Figure 9.

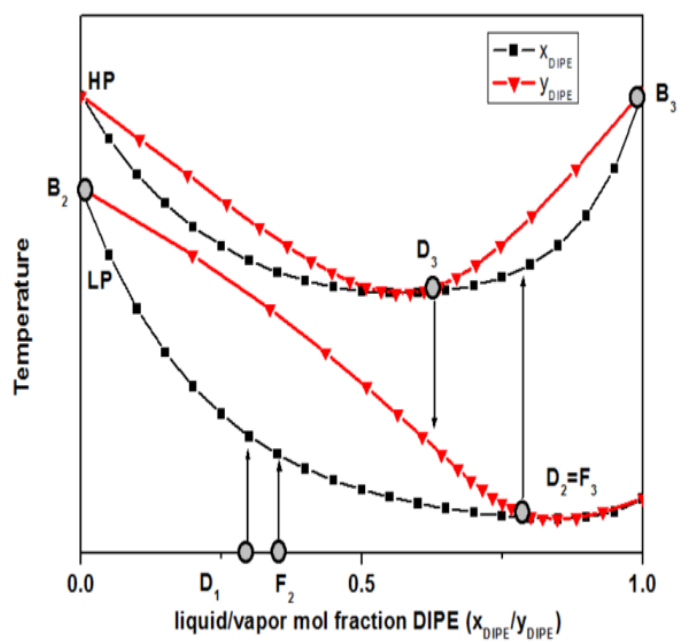

Figure 7: Txy for DIPE(1)+IPA(2) azeotropic mixture and distillation sequence

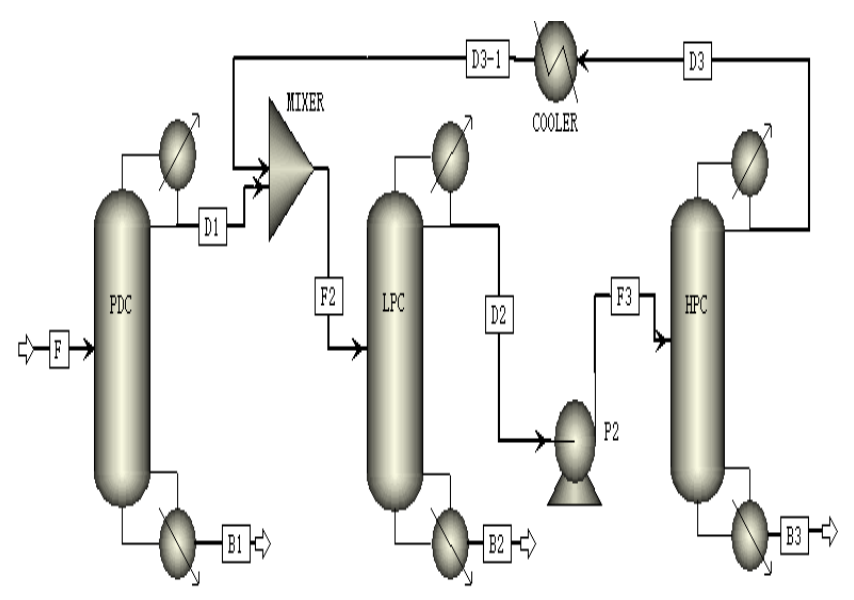

Figure 8: Separation process of DIPE(1)+IPA(2)+NPA(3) by PSD

\subsubsection{Economic evaluation}

In order to compare the PSD process with the ED process, it will be necessary to evaluate economically the PSD optimum, that is, calculate the total annual costs (TAC). Details of the columns for PSD and ED process together with the total annual costs (TAC) for optimum ED-3 and PSD-2 case are listed in Table 1 and Figure 9 . As can be seen, the annual costs estimated for the PSD process $\left(12.62 \times 10^{5} \$\right)$ are slightly lower than the ones corresponding to the ED process $\left(12.93 \times 10^{5} \$\right)$ both obtained using the same criteria and evaluation procedures. Capital investment of PSD process is little higher than that of ED process. If we consider larger plants, the difference between both alternatives becomes less and less, precisely because the steam costs grow almost proportionally to the flow rate, while the costs associated with the investment grow much more slowly, and therefore the total costs of both alternatives are much closer.

Extractive distillation has the inherent disadvantage of introducing a third component into the system (the solvent) that will appear in the product streams, which necessarily have to be recovered. So, the extractive system must show significant economic advantages over the pressure-swing system to make it the process of choice.

\section{CONCLUSIONS}

In this case, steady-state comparisons have been presented of a pressureswing distillation process and an extractive distillation process to separate the DIPE+IPA+NPA, azeotropic mixture. The computer simulation and economic evaluation of the two separation alternatives allow us to conclude that, to process $100 \mathrm{kmol} / \mathrm{h}$, the process that uses PSD is more attractive, although the TAC of two process are similar to each other. 


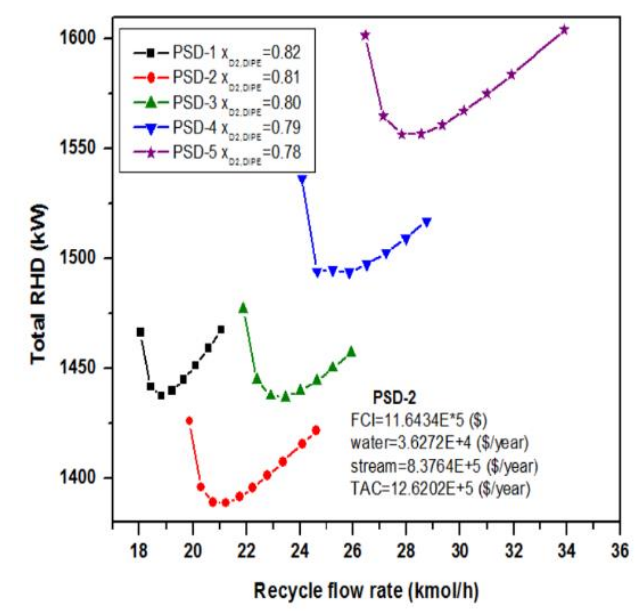

Figure 9: Total reboiler heat duty vs. azeotropic composition (XD2,DIPE) of LPC in PSD

\section{REFERENCES}

[1] Lladosa, E., Montón, J.B., Burguet, M.C., Dela Torre, J. 2008. Isobaric (vapour + liquid + liquid) equilibrium data for (di-n-propyl ether $+n$ propyl alcohol + water) and (diisopropylether + isopropyl alcohol + water) systems at $100 \mathrm{kPa}$. The Journal of Chemical Thermodynamics, 40, 867873.

[2] Lladosa, E., Montón, J.B., Burguet, M.C., Munoz, R. 2007. Effect of pressure and the capability of 2-methoxyethanol as a solventin the behaviour of a diisopropyl ether-isopropyl alcoholazeotropic mixture. Fluid Phase Equilibria, 262, 271-279.

[3] Zhongmin, L., Shukui, Z., Shigang, Z., Hongyi, Y. 2005. Progress on Production of Isopropanolby Direct Hydration of Propylene [J]. Fine and Specialty Chemicals, 13 (15), 1-4.

[4] Manan, Z.A., Bañares-Alcántera, R. 2001. A New Catalog of the Most Promising Separation Sequences for Homogeneous Azeotropic Mixtures I. Systems without Boundary Crossing. Industrial \& Engineering Chemistry Research, 40, 5795-5809.

[5] Luo, H., Liang, K., Li, W., Li, Y., Xia, M., Xu, C. 2014. Comparison of pressure-swing distillation and extractive distillation methods for isopropyl alcohol/diisopropyl ether separation. Industrial \& Engineering Chemistry Research, 53, 15167-15182.

[6] Biegler, L.T., Grossmann, I.E., Westerberg, A.W., Bieglerc, L.T. 1997. Systematic Methods of Chemical Process Design, Pearson Schweiz Ag.

[7] Serafimov, L. 1996. Thermodynamic and topological analysis of liquidvapor phase equilibrium diagrams and problems of rectification of multicomponent mixtures. Mathematical Methods in Contemporary Chemistry.

[8] Lei, Z., Li, C., Chen, B. 2003. Extractive distillation: a review. Separation and Purification Technology, 32,121-213. 